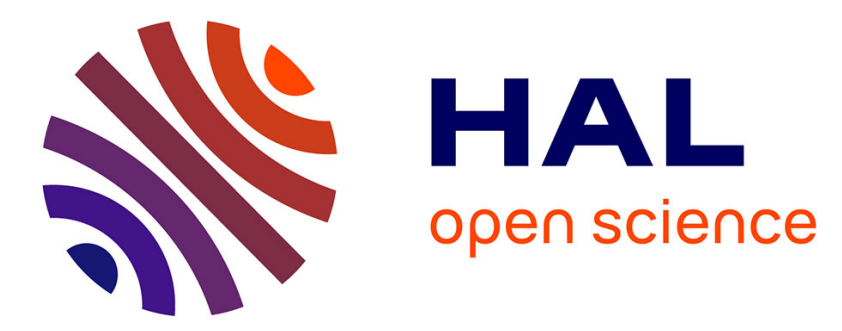

\title{
Composition chimique des dépôts atmosphériques à l'horizon 2020-2040
}

Aude Pascaud, Stéphane Sauvage, Christian Pagé, Olivier Roustant, Anne Probst, Manuel Nicolas, Luc Croisé, Abdelkrim Mezdour, Patrice Coddeville

\section{- To cite this version:}

Aude Pascaud, Stéphane Sauvage, Christian Pagé, Olivier Roustant, Anne Probst, et al.. Composition chimique des dépôts atmosphériques à l'horizon 2020-2040. La Météorologie, 2016, $\mathrm{n}^{\circ}$ 92, pp.56-65. $10.4267 / 2042 / 58223$. hal-01280712

\section{HAL Id: hal-01280712 \\ https://hal.science/hal-01280712}

Submitted on 1 Mar 2016

HAL is a multi-disciplinary open access archive for the deposit and dissemination of scientific research documents, whether they are published or not. The documents may come from teaching and research institutions in France or abroad, or from public or private research centers.
L'archive ouverte pluridisciplinaire HAL, est destinée au dépôt et à la diffusion de documents scientifiques de niveau recherche, publiés ou non, émanant des établissements d'enseignement et de recherche français ou étrangers, des laboratoires publics ou privés. 


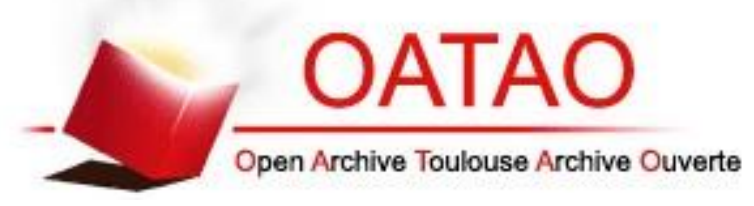

\section{Open Archive TOULOUSE Archive Ouverte (OATAO)}

OATAO is an open access repository that collects the work of Toulouse researchers and makes it freely available over the web where possible.

This is an author-deposited version published in : http://oatao.univ-toulouse.fr/ Eprints ID : 15123

To link to this article : DOI : $10.4267 / 2042 / 58223$

URL : http://dx.doi.org/10.4267/2042/58223

To cite this version : Pascaud, Aude and Sauvage, Stéphane and Pagé, Christian and Roustant, Olivier and Probst, Anne and Nicolas, Manuel and Croisé, Luc and Mezdour, Abdelkrim and Coddeville, Patrice Composition chimique des dépôts atmosphériques à l'horizon 20202040. (2016) La Météorologie ( $\left.{ }^{\circ} 92\right)$. pp.59-65. ISSN 0026-1181

Any correspondance concerning this service should be sent to the repository administrator: staff-oatao@ listes-diff.inp-toulouse.fr 


\section{Composition chimique des dépôts atmosphériques à I'horizon 2020-2040}

\section{Résumé}

Les dépôts atmosphériques peuvent causer des dommages importants aux milieux naturels. Des mesures à long terme issues de la surveillance des dépôts atmosphériques en France ont été exploitées pour construire un modèle statistique prévisionnel afin d'estimer les changements de la composition chimique des dépôts atmosphériques à l'horizon 2020 2040. A partir de simulations d'évolutions possibles du climat et des émissions de polluants atmosphériques, les dépôts de sulfate non marin et les dépôts d'ammonium sont susceptibles de diminuer, mais ceux de nitrate pourraient augmenter de façons diverses selon les régions. Le changement des variations saisonnières de pluviométrie pourrait augmenter les flux de dépôts.

\section{Abstract}

\section{Chemical composition of atmospheric deposition for the period 2020-2040}

Atmospheric deposition can have harmful effect on the ecosystems. Forecast statistical models were based on long-term monitoring data of chemical composition measured in France. Using climate and pollutant emissions simulations, the projected changes in chemical composition of atmospheric deposition were analyzed for the period 2020-2040: sulfate and ammonium deposition fluxes are likely to decrease but nitrate deposition fluxes might increase with some regional discrepancies. Projected changes in precipitation seasonal variations are likely to drive an increase in deposition fluxes.

Aude Pascaud 1 , Stéphane Sauvage ${ }^{1}$, Christian Pagé2, Olivier Roustant ${ }^{3}$, Anne Probst ${ }^{4}$, Manuel Nicolas ${ }^{5}$, Luc Croisé ${ }^{5}$, Abdelkrim Mezdour 6 , Patrice Coddeville ${ }^{1}$

1 Département Sciences de l'atmosphère et génie de l'environnement, Mines Douai, Douai

2 Sciences de l'Univers au Cerfacs, Cerfacs / CNRS, Toulouse

3 Laboratoire d'informatique, de modélisation et d'optimisation des systèmes, Mines Saint-Étienne / CNRS, Saint-Étienne

4 Laboratoire écologie fonctionnelle et environnement, CNRS / Université de Toulouse / Institut national polytechnique de Toulouse, Castanet-Tolosan

5 Direction technique et commerciale bois, département recherche et développement, Office national des forêts, Fontainebleau

6 Direction interrégionale Nord, Météo-France, Villeneuve d'Ascq

aude.pascaud@mines-douai.fr

es composés atmosphériques, qu'ils soient de sources naturelles ou anthropiques, peuvent être transformés, transportés sur de longues distances et transférés de l'atmosphère vers un autre réservoir par dépôts atmosphériques. Ces composés peuvent générer des événements de pollution dont la prévision est utile à la protection de la santé publique et des milieux naturels (de Vries et Posch, 2011). Au cours des deux dernières décennies, des mesures ont été prises afin de limiter les effets des émissions polluantes sur le climat, la santé humaine et les écosystèmes. L'efficacité de ces mesures peut être évaluée par le suivi à long terme de la chimie des dépôts atmosphériques (Vet et al., 2014).

Les futures politiques de réduction des émissions polluantes nécessitent l'étude conjointe de scénarios prédictifs des activités polluantes, du climat et des impacts environnementaux (Schucht et al., 2015). Intégrant les effets climatiques et selon les connaissances actuelles, les modèles de chimie-transport sont en mesure de prévoir le devenir et les évolutions des concentrations de polluants dans l'atmosphère et d'en déduire, de manière spatialisée et temporelle, leurs dépôts sur les écosystèmes (de Vos et Zhang, 2012). Cependant, ces modèles déterministes intègrent des systèmes physicochimiques complexes, qui demandent une connaissance détaillée des émissions, des processus, de la climatologie et de l'occupation des sols. Des campagnes d'observations intensives multiparamètres sont souvent organisées afin d'améliorer les processus physiques et chimiques pris en compte dans les modèles physiques. Une approche complémentaire pour prévoir les polluants atmosphériques consiste à réaliser une analyse statistique à partir de longues séries d'observations. Il s'agit d'abord d'établir des modèles statistiques à partir d'indicateurs statistiques, représentant de manière compréhensible les caractéristiques intrinsèques du jeu de données et reproduisant les composés polluants. La modélisation statistique permet de fournir des évolutions possibles d'observations en un point et en un temps donnés. Ces modèles statistiques sont simples à mettre en œuvre, mais ils sont limités en matière de représentativité spatiale et dépendants de l'incertitude des mesures.

En France, des observatoires nationaux sont dédiés à la surveillance des dépôts atmosphériques depuis le début des années 1990. Parmi les éléments majeurs inorganiques, les ions sulfate non marin $\left(\mathrm{nssSO}{ }_{4}^{2-}\right)$, nitrate $\left(\mathrm{NO}_{3}^{-}\right)$et ammonium $\left(\mathrm{NH}_{4}^{+}\right)$sont particulièrement suivis pour leurs impacts acidifiant et eutrophisant sur les écosystèmes. Ces ions solubilisés sont issus de la transformation des gaz précurseurs 
$\mathrm{SO}_{x}, \mathrm{NO}_{x}$ et $\mathrm{NH}_{3}$ émis majoritairement par les activités anthropiques. La mutualisation des mesures de ces observatoires offre un jeu de données inédit pour étudier l'évolution de la composition chimique des dépôts atmosphériques au niveau national. À partir de ces données, cette étude vise à construire un modèle statistique prévisionnel, puis à simuler les changements projetés de la composition chimique des dépôts à l'horizon 2020-2040 suivant plusieurs simulations d'évolutions du climat et des émissions polluantes. Cette étude a pour vocation de s'assurer que les politiques publiques de réductions d'émissions ont des conséquences directes sur la qualité de l'air. L'évaluation de l'efficacité des politiques se restreint ici à s'assurer que la réduction est effectivement observée.

\section{Observatoires nationaux des dépôts atmosphériques}

Sur le territoire métropolitain français, trois observatoires ont en commun la surveillance de la qualité de l'air en zone rurale, dont notamment la mesure des dépôts atmosphériques et la mesure de paramètres météorologiques (pluviométrie) (tableau 1).

L'observatoire Bapmon (programme Veille atmosphérique globale (VAG) de l'Organisation mondiale de la météorologie) a été mis en place en France à partir de 1977 par Météo-France (Cénac et Zéphoris, 1992). Il permet d'améliorer la compréhension du comportement de l'atmosphère à l'échelle globale et ses interactions avec les océans et la biosphère (Kohler, 1980). Jusqu'à la fin de leur suivi en 2007, les mesures sur trois stations de cet observatoire étaient réalisées à partir de collectes hebdomadaires de dépôts humides. Suite à un audit en 2008, les trois sites ont été fermés pour être remplacés par des sites équivalents de l'observatoire Mera/Emep et labellisés par le programme VAG.

L'observatoire Mera, créé en 1984 dans le cadre de la convention de Genève sur la pollution atmosphérique transfrontière à longue distance, constitue la contribution française à l'Emep (programme concerté de surveillance et d'évaluation du transport à longue distance des polluants atmosphériques

Tableau 1. Descriptif et périodes d'observations des stations de surveillance de la qualité de l'air en zone rurale sur le territoire métropolitain français.

\begin{tabular}{|c|c|c|c|c|c|c|}
\hline Observations & Station & Acronyme & Période d'observation & Altitude (m) & Latitude (deg min) & Longitude (deg min) \\
\hline BAPMON & Abbeville & WB80AB & 1990-2007 & 70 & $50^{\circ} 08^{\prime} \mathrm{N}$ & $01^{\circ} 50^{\prime} \mathrm{E}$ \\
\hline BAPMON & Carpentras & WB84CA & 1990-2007 & 99 & $44^{\circ} 05^{\prime} \mathrm{N}$ & $05^{\circ} 03^{\prime} \mathrm{E}$ \\
\hline BAPMON & Gourdon & WB46G0 & 1990-2007 & 259 & $44^{\circ} 45^{\prime} \mathrm{N}$ & $01^{\circ} 24^{\prime} \mathrm{E}$ \\
\hline MERA & Brotonne & WM76BR & 1990-2007 & 115 & $49^{\circ} 24^{\prime} \mathrm{N}$ & $00^{\circ} 42^{\prime} \mathrm{E}$ \\
\hline MERA & Le Casset & WM04CA & 1990-2008 & 1750 & $45^{\circ} 00^{\prime} \mathrm{N}$ & $06^{\circ} 28^{\prime} \mathrm{E}$ \\
\hline MERA & Donon & WM67D0 & $1990-2008$ & 775 & $48^{\circ} 30^{\prime} \mathrm{N}$ & $01^{\circ} 50^{\prime} \mathrm{E}$ \\
\hline MERA & Iraty & WM64IR & 1990-2008 & 1300 & $43^{\circ} 02^{\prime} N$ & $01^{\circ} 50^{\prime} \mathrm{W}$ \\
\hline MERA & Morvan & WM58M0 & 1990-2008 & 620 & $47^{\circ} 16^{\prime} \mathrm{N}$ & $04^{\circ} 05^{\prime} \mathrm{E}$ \\
\hline MERA & Peyrusse Vieille & WM32PE & 1995-2008 & 236 & $43^{\circ} 37^{\prime} \mathrm{N}$ & $00^{\circ} 11^{\prime} \mathrm{E}$ \\
\hline MERA & Revin & WM08RE & 1990-2008 & 390 & $49^{\circ} 54^{\prime} \mathrm{N}$ & $04^{\circ} 38^{\prime} \mathrm{E}$ \\
\hline CATAENAT & СНP4OHCT & $\mathrm{BC} 40 \mathrm{~GB}$ & 1993-2008 & 20 & $43^{\circ} 44^{\prime} \mathrm{N}$ & $00^{\circ} 50^{\prime} \mathrm{W}$ \\
\hline CATAENAT & СНР59НСТ & BC59L0 & 1993-2008 & 149 & $50^{\circ} 10^{\prime} \mathrm{N}$ & $03^{\circ} 45^{\prime} \mathrm{E}$ \\
\hline CATAENAT & CHS35HCT & BC35LI & 1993-2007 & 80 & $48^{\circ} 10^{\prime} \mathrm{N}$ & $01^{\circ} 32^{\prime} \mathrm{W}$ \\
\hline CATAENAT & CHS41HCT & BC41CC & 1993-2008 & 127 & $47^{\circ} 35^{\prime} \mathrm{N}$ & $01^{\circ} 15^{\prime} \mathrm{E}$ \\
\hline CATAENAT & CPS77HCT & BC77F0 & 1993-2008 & 80 & $48^{\circ} 27^{\prime} \mathrm{N}$ & $02^{\circ} 43^{\prime} \mathrm{E}$ \\
\hline CATAENAT & DOU71HCT & BC71AN & 1993-2008 & 650 & $47^{\circ} 04^{\prime} \mathrm{N}$ & $04^{\circ} 05^{\prime} \mathrm{E}$ \\
\hline CATAENAT & ЕРСОВНСТ & ВС08TH & 1993-2008 & 480 & $49^{\circ} 55^{\prime} \mathrm{N}$ & $04^{\circ} 48^{\prime} \mathrm{E}$ \\
\hline CATAENAT & ЕРС63НСТ & BC63GC & 1993-2008 & 950 & $45^{\circ} 45^{\prime} \mathrm{N}$ & $02^{\circ} 57^{\prime} \mathrm{E}$ \\
\hline CATAENAT & ЕРC74HCT & BC47CE & 1993-2008 & 1200 & $46^{\circ} 13^{\prime} \mathrm{N}$ & $06^{\circ} 20^{\prime} \mathrm{E}$ \\
\hline CATAENAT & ЕРС87НCT & BC87PC & 1993-2008 & 650 & $45^{\circ} 47^{\prime} \mathrm{N}$ & $01^{\circ} 49^{\prime} \mathrm{E}$ \\
\hline CATAENAT & НЕТЗОНСТ & BC30VA & 1993-2008 & 1400 & $44^{\circ} 06^{\prime} \mathrm{N}$ & $03^{\circ} 32^{\prime} \mathrm{E}$ \\
\hline CATAENAT & HET54HCT & BC54AZ & 1993-2007 & 325 & $48^{\circ} 29^{\prime} \mathrm{N}$ & $06^{\circ} 42^{\prime} \mathrm{E}$ \\
\hline CATAENAT & HET64HCT & BC64AN & 1993-2008 & 400 & $43^{\circ} 08^{\prime} \mathrm{N}$ & $00^{\circ} 40^{\prime} \mathrm{W}$ \\
\hline CATAENAT & PL2OHCT & BC20EV & 1993-2007 & 1100 & $42^{\circ} 15^{\prime} \mathrm{N}$ & $08^{\circ} 50^{\prime} \mathrm{E}$ \\
\hline CATAENAT & PM17HCT & $\mathrm{BC} 17 \mathrm{GO}$ & 1993-2008 & 15 & $45^{\circ} 58^{\prime} \mathrm{N}$ & $01^{\circ} 16^{\prime} \mathrm{W}$ \\
\hline CATAENAT & PM40HCT & BC4OLO & 1993-2008 & 150 & $44^{\circ} 02^{\prime} \mathrm{N}$ & $00^{\circ} 00^{\prime} \mathrm{W}$ \\
\hline CATAENAT & PM72HCT & BC72LA & 1993-2008 & 153 & $47^{\circ} 48^{\prime} \mathrm{N}$ & $00^{\circ} 21^{\prime} \mathrm{E}$ \\
\hline CATAENAT & РM85HCT & BC85DM & 1993-2008 & 75 & $46^{\circ} 50^{\prime} \mathrm{N}$ & $02^{\circ} 08^{\prime} \mathrm{W}$ \\
\hline CATAENAT & PS44HCT & BC44GA & 1993-2008 & 38 & $47^{\circ} 33^{\prime} \mathrm{N}$ & $01^{\circ} 45^{\prime} \mathrm{W}$ \\
\hline CATAENAT & PS67HCT & BC67HA & 1993-2008 & 175 & $48^{\circ} 50^{\prime} \mathrm{N}$ & $07^{\circ} 43^{\prime} \mathrm{E}$ \\
\hline CATAENAT & PS76HCT & BC76MS & 1993-2008 & 70 & $49^{\circ} 27^{\prime} \mathrm{N}$ & $00^{\circ} 43^{\prime} \mathrm{E}$ \\
\hline CATAENAT & PS05HCT & BC05CR & 1993-2008 & 1360 & $44^{\circ} 29^{\prime} \mathrm{N}$ & $06^{\circ} 27^{\prime} \mathrm{E}$ \\
\hline CATAENAT & SP11HCT & $\mathrm{BC} 11 \mathrm{BE}$ & 1993-2007 & 950 & $42^{\circ} 52^{\prime} \mathrm{N}$ & $02^{\circ} 04^{\prime} \mathrm{E}$ \\
\hline CATAENAT & SP25HCT & ВС25M0 & 1993-2008 & 1000 & $46^{\circ} 58^{\prime} \mathrm{N}$ & $06^{\circ} 27^{\prime} \mathrm{E}$ \\
\hline CATAENAT & SP38HCT & ВС38СВ & 1993-2008 & 1100 & $45^{\circ} 25^{\prime} \mathrm{N}$ & $06^{\circ} 07^{\prime} \mathrm{E}$ \\
\hline CATAENAT & SP57HCT & BC57AB & 1993-2008 & 400 & $48^{\circ} 35^{\prime} \mathrm{N}$ & $07^{\circ} 09^{\prime} \mathrm{E}$ \\
\hline CATAENAT & SP68HCT & BC68LA & 1993-2008 & 680 & $47^{\circ} 55^{\prime} \mathrm{N}$ & $07^{\circ} 07^{\prime} \mathrm{E}$ \\
\hline
\end{tabular}




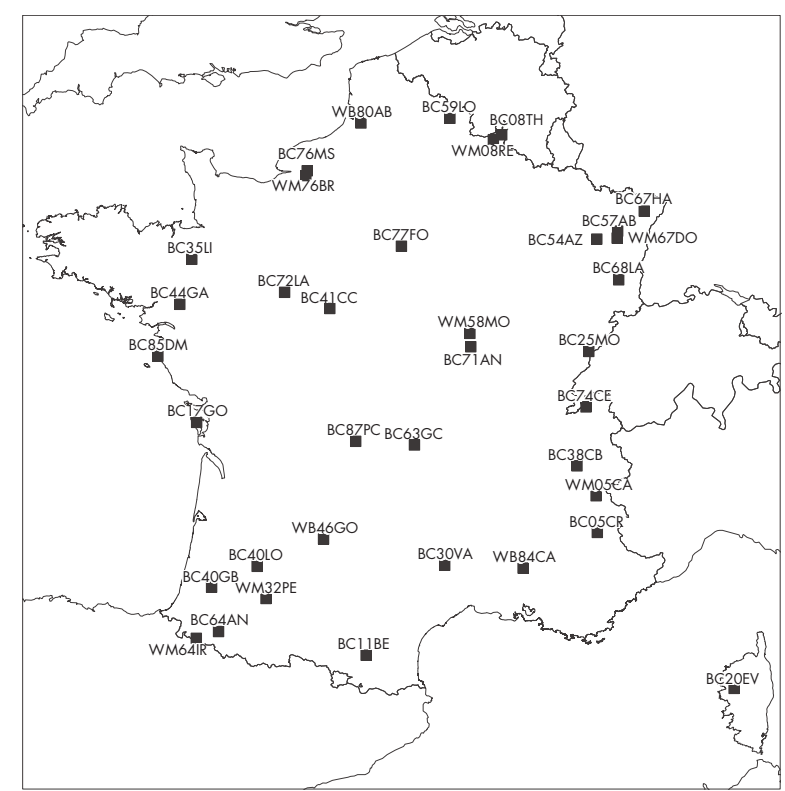

Figure 1. Localisation des stations de mesure des dépôts atmosphériques en zone rurale sur le territoire métropolitain français.

en Europe). Il permet d'étudier l'évolution des problématiques d'acidification, d'eutrophisation, de pollution photooxydante et particulaire (Sauvage et al., 2009). La mesure des dépôts atmosphériques humides est réalisée avec une fréquence journalière sur sept stations couvrant la période 1990-2008.

L'observatoire Cataenat a été mis en place par l'Office national des forêts à la suite des recherches menées dans le cadre du programme de recherche Deforpa (Muller, 1984 ; Landmann, 1991). Implanté à partir de 1992 sur une partie des sites du réseau français Renecofor contribuant à l'ICP Forests (programme international concerté sur l'évaluation et la surveillance des effets de la pollution atmosphérique sur les forêts), il permet de suivre l'état de santé des écosystèmes forestiers à l'échelle de la France métropolitaine en réponse notamment aux pollutions atmosphériques (Barthod, 1994). Les dépôts atmosphériques totaux sont collectés à une fréquence hebdomadaire en zone de clairière à proximité de 27 stations forestières. Les analyses sont réalisées sur des échantillons composites par période de quatre semaines (Ulrich et al., 1998).

Les concentrations de composés inorganiques dans les dépôts mesurées dans ces stations sont représentatives des concentrations présentes naturellement ou liées à des apports transfrontaliers issus de la dispersion de polluants émis par des sources d'émissions lointaines. La période étudiée pour les trois réseaux est comprise entre 1990 et 2008. Au total, les 37 stations sont réparties de manière homogène sur le territoire métropolitain (figure 1) et sont représentatives de différentes zones climatiques. Les analyses concernent le $\mathrm{pH}$, les principaux anions $\left(\mathrm{Cl}^{-}\right.$, $\left.\mathrm{NO}_{3}^{-}, \mathrm{SO}_{4}^{2-}\right)$ et cations $\left(\mathrm{Na}^{+}, \mathrm{K}^{+}, \mathrm{Mg}^{2+}\right.$, $\left.\mathrm{Ca}^{2+}, \mathrm{NH}_{4}^{+}\right)$. Les dépôts ioniques journaliers sont calculés à partir du produit de la pluviométrie journalière mesurée sur les stations et des concentrations ioniques journalières des échantillons de pluie collectés. Les analyses chimiques ont été réalisées par le même laboratoire, régulièrement impliqué dans des tests de comparaison interlaboratoires internationaux (Uggerud et Hjellbrekke, 2011).

\section{Modélisation des dépôts atmosphériques}

La prévision de la composition chimique des dépôts atmosphériques a donné lieu à l'utilisation de nombreuses techniques statistiques dans la littérature. Par exemple, des techniques de modélisation par régression linéaire (Moustris et al., 2012), non linéaire (Chattopadhyay et al., 2012) ou par analyse de Box-Jenkins (Nickerson et Les techniques statistiques de prévision sont présentées en détail dans Makridakis et al. (1998). Dans notre étude, des variables explicatives vont servir à prévoir les variations des dépôts de $\mathrm{nssSO}_{4}^{2-}, \mathrm{NO}_{3}^{-}$et $\mathrm{NH}_{4}^{+}$. À terme, l'objectif est de prévoir les variations, dans un avenir proche, des dépôts de ces mêmes ions. Pour la suite de la présentation de la méthodologie, il sera pris pour exemple les valeurs de dépôts Madsen, 2005) ont pu être appliquées. mensuels de $\mathrm{nssSO}_{4}^{2-}$ au site WM08RE (voir tableau 1). La totalité de la procédure a ensuite été appliquée aux dépôts de $\mathrm{nssSO}_{4}^{2-}, \mathrm{NO}_{3}^{-}$et $\mathrm{NH}_{4}^{+}$sur les sites des trois observatoires.

\section{Choix des variables explicatives}

À la vue des contraintes de prévisions, les valeurs mesurées de dépôts mensuels de $\mathrm{nsSO}_{4}^{2-}, \mathrm{NO}_{3}^{-}$et $\mathrm{NH}_{4}^{+}$, exprimées en équivalent par hectare $\left(\mathrm{eq} \cdot \mathrm{ha}^{-1}\right)^{1}$, ont été ajustées avec les variables suivantes.

Concernant les émissions polluantes pour l'Union européenne des 27 (UE27), les données annuelles d'émissions (exprimées en téragrammes, $\mathrm{Tg}$ ) sont issues du Centre sur les inventaires des émissions et projections (EMEP/CEIP, 2012). Les émissions ont été interpolées linéairement à l'échelle mensuelle sur la période 1990-2008. Avec ce choix, le modèle ne prend pas en compte l'impact potentiel des émissions hors Europe, sachant que le projet Hemispheric Transport of Air Pollution (Carmichael et Wild, 2010) suggère qu'elles peuvent contribuer jusqu'à $20 \%$ de la pollution européenne.

Les données mensuelles (12 mois par an pour les observatoires Mera et 13 périodes de quatre semaines par an pour l'observatoire Cataenat) de précipitations totales (notées $P l$ et exprimées en millimètre) sont issues de l'analyse Safran. Safran est une ré-analyse météorologique proche de la surface et à méso-échelle développée par MétéoFrance (Quintana-Segui et al., 2008). Utilisées sur la période 1990-2008, les précipitations totales mensuelles sont considérées dans la maille la plus proche de la station.

Pour reproduire les variabilités saisonnières des émissions polluantes, de la dynamique atmosphérique et de la capacité oxydante de l'atmosphère, il a été choisi d'utiliser des indices saisonniers $[-1 ; 1]$, basés sur la première harmonique d'une série de Fourier de période annuelle. Ils correspondent aux fonctions $\cos (\omega t)$ et $\sin (\omega t)$ avec $t$ variable de temps et $\omega=2 \pi / T$, où $T=12$ pour les observatoires Mera et Bapmon

1. Par praticité, l'unité utilisée est "l'équivalent» (eq) par hectare au lieu de l'unité du Système international, la quantité de matière (mol) par mètre carré. Cela correspond au produit du nombre de mol par le nombre de charges de même signe portées par l'ion. 
et $T=13$ pour l'observatoire Cataenat. Ces indices sont notés dans le texte sais.cos pour $\cos (\omega t)$ et sais.sin pour $\sin (\omega t)$.

\section{Forme générale du modèle}

Pour prévoir les tendances des dépôts, la régression linéaire multiple a été choisie pour plusieurs raisons. Tout d'abord, car ce type de modèle statistique se construit pas à pas afin de comprendre les évolutions de la série temporelle étudiée (Makridakis et al., 1998). Ensuite, car la principale et la plus significative relation que l'on observe entre les variables explicatives prédéfinies plus haut est linéaire (figure 2). En effet, la corrélation entre le logarithme des dépôts de $\mathrm{nsSSO}_{4}^{2-}$ au site WM08RE sur la période 1990-2008 et le logarithme des précipitations totales mensuelles est significative et égale à 0,65 . Ce résultat est explicable, car le dépôt est principalement humide et augmente avec la hauteur des précipitations. En revanche, les émissions mensuelles extrapolées linéairement ont une corrélation faible et non significative $(0,11)$ avec le logarithme des dépôts mensuels. Bien que significative, la corrélation entre $\log _{10}(P l)$ et sais.cos n'est pas forte et n'a pas été une source de colinéarité lors de l'estimation des coefficients. Pour être complet, le diagnostic des interactions entre les variables explicatives a été réalisé et il n'a pas été observé d'interactions dans les jeux de données. L'hypothèse de fond est que les caractéristiques historiques des données seront les mêmes dans l'avenir.

La forme générale de ce type de modèle par régression linéaire multiple est :

$Y=\beta_{0}+\beta_{1} \times X_{1}+\ldots+\beta_{k} \times X_{k}+\varepsilon$

où $Y$ est la variable expliquée ; $X_{1}, \ldots$, $X_{k}$ les variables explicatives $; \varepsilon$ le terme d'erreur aléatoire de distribution normale (espérance nulle et variance constante) ; $\beta_{0}$ et $\beta_{k}$ les paramètres constants de la régression. La forme de la régression linéaire multiple entre $Y$ et ses variables explicatives devient par exemple, pour le cas des dépôts mensuels de sulfate non marin au site WM08RE sur la période 1990-2008 :

$\log _{10}\left(\mathrm{nssSO}_{4}^{2-}\right)_{i}=0,001+0,002 \times \mathrm{SO}_{x i}+$ $0,644 \times \log _{10}(P l)_{i}-0,071 \times$ sais. $\cos _{i}$ $+0,013 \times$ sais.sin $_{i}+\varepsilon_{i}$

La figure 3 représente l'évolution temporelle des dépôts mensuels de $\mathrm{nssSO}_{4}^{2-}$ comparée à celle d'une influence climatique dans le modèle de régression. Cette contribution climatique se

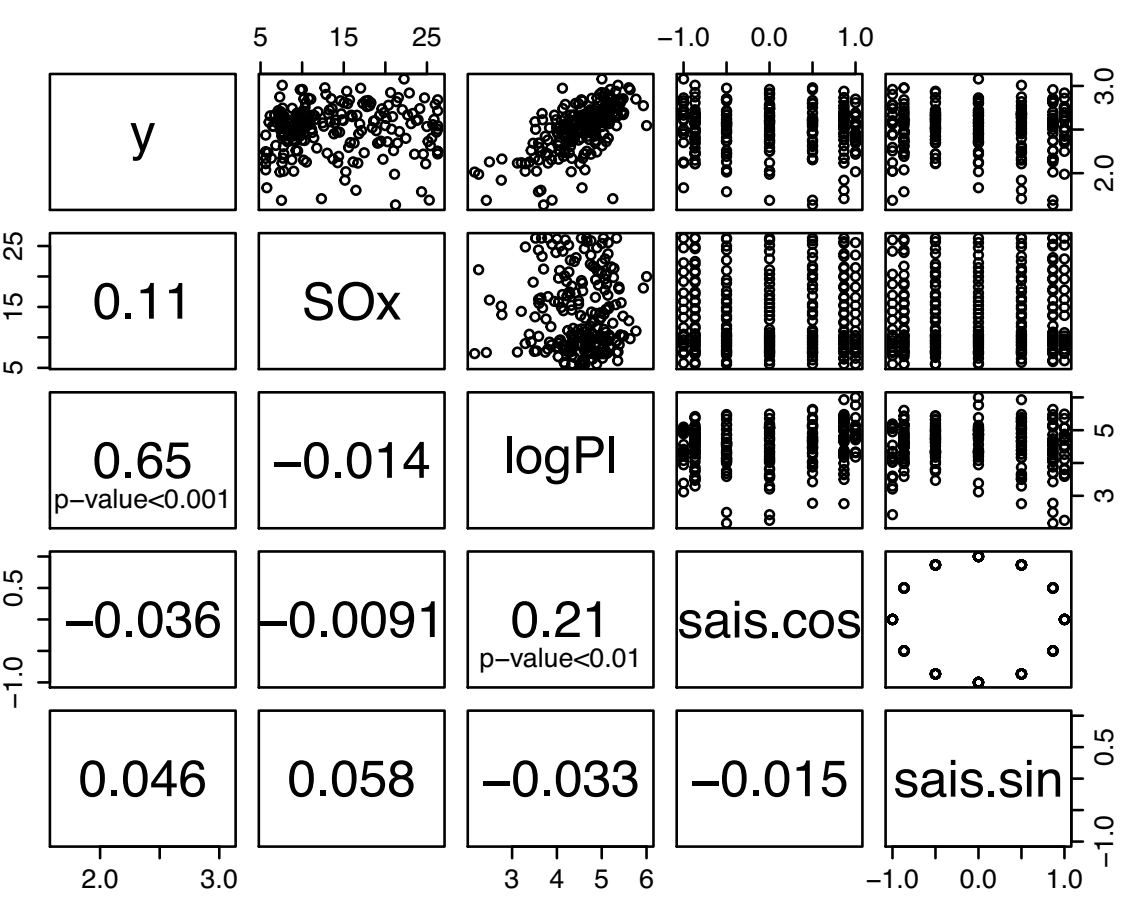

Figure 2. Évaluation de l'existence de liens entre les variables explicatives $\left(\mathrm{SO}_{x}, \log _{10} P I\right.$, sais. cos et sais.sin) et le logarithme des dépôts de $\mathrm{nsSSO}_{4}^{2-}(y)$ au site WM08RE sur la période 1990-2008. Cette matrice des corrélations (test de Spearman basé sur les rangs) présente sur le panneau de droite les régressions bivariées et sur le panneau de gauche la valeur de la corrélation et sa significativité statistique.

compose de l'ordonnée à l'origine, de la variable météorologique $(P l)$ et des variables de saisonnalité (sais.cos et sais.sin) (équation 2). Elle reproduit les variations fréquentes des dépôts mensuels de nssSO ${ }_{4}^{2-}$. Pour se rendre compte de ce qui n'est pas expliqué par la part climatique modélisée, la fonction yspec a été calculée comme la différence entre les dépôts mensuels de $\mathrm{nsSSO}_{4}^{2-}$ et la part climatique modélisée (figure 4). La tendance de la fonction yspec, obtenue par lissage, présente

une structure proche de celle d'une part appelée anthropique. Cette part anthropique est modélisée dans la figure 4 par la variable explicative des émissions de dioxyde de soufre dans l'Union Européenne multipliée par la constante 0,002 (équation 2). Ces émissions de dioxyde de soufre ont diminué en Europe depuis 1990. Cette tendance est bien présente dans la fonction yspec, ce qui confirme que les dépôts de $\mathrm{nssSO}_{4}^{2-}$ ont diminué depuis 1990 sur le site WM08RE.

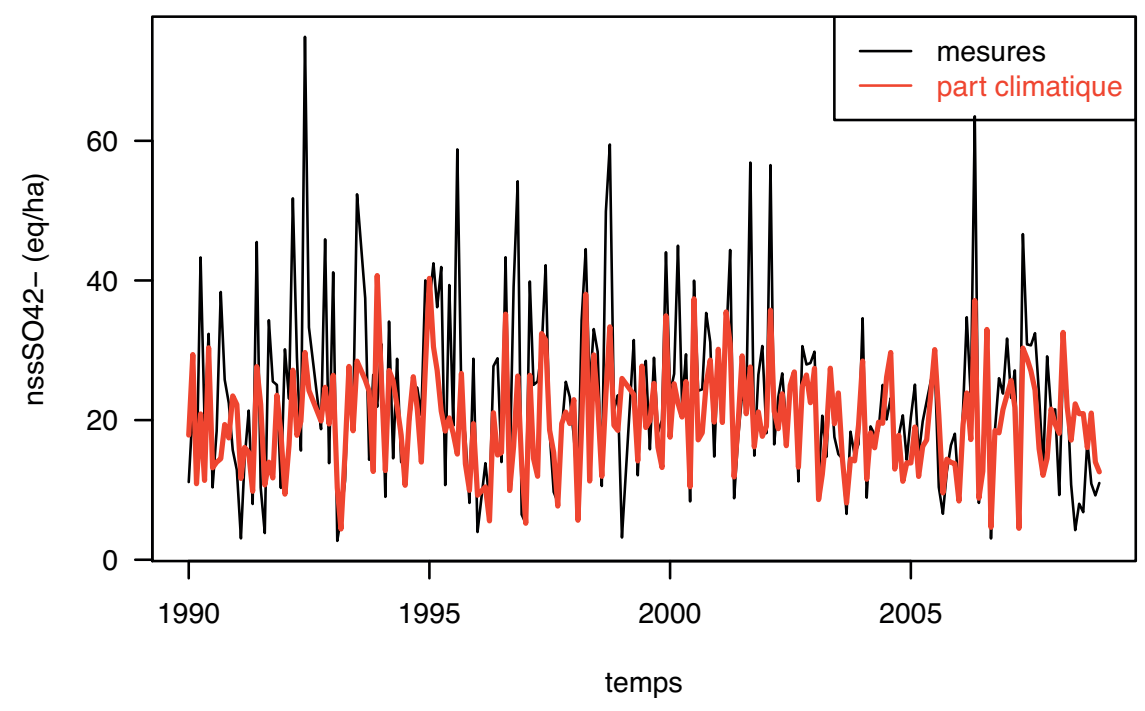

Figure 3. Évolutions temporelles des dépôts mensuels (en noir) de sulfate non marin au site WM08RE sur la période 1990-2008 et de la part climatique modélisée (en rouge). 


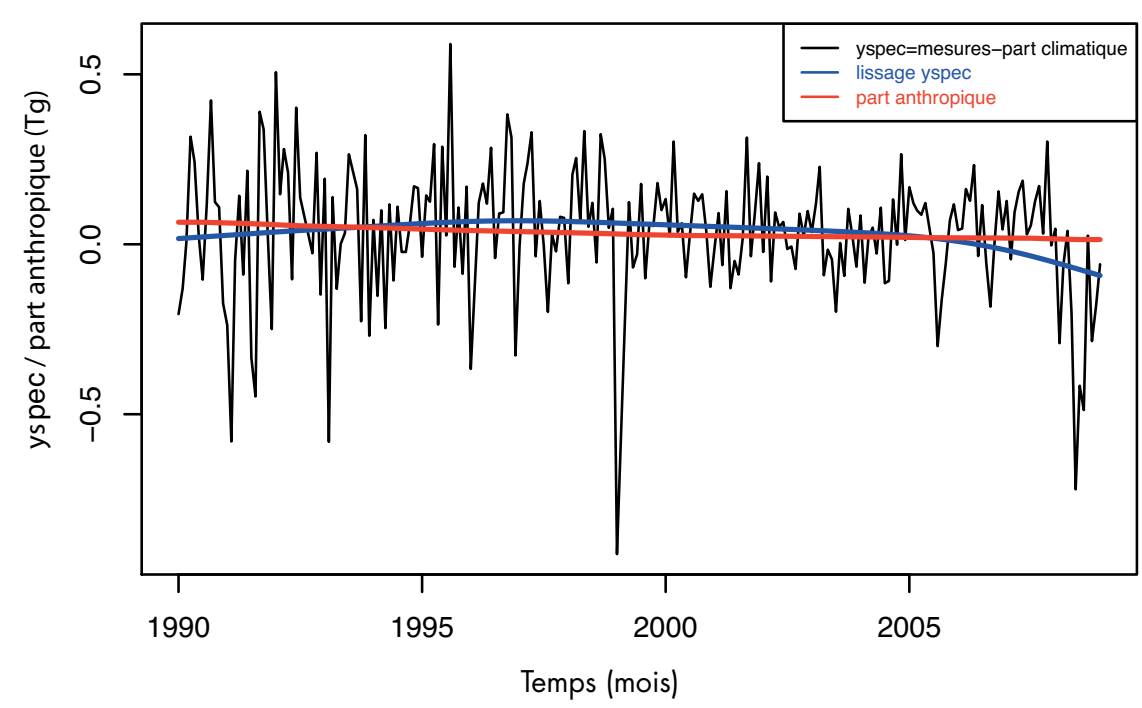

Figure 4. Comparaison des distributions de yspec (sans unité, en noir), de la droite de lissage sur yspec (en bleu) et de la part anthropique modélisée (en Tg, en rouge) pour les dépôts mensuels de sulfate non marin au site WM08RE sur la période 1990-2008.

\section{Protocole de validation}

Une analyse des résidus a ensuite été appliquée aux dépôts de nss $\mathrm{SO}_{4}^{2-}, \mathrm{NO}_{3}^{-}$ et $\mathrm{NH}_{4}^{+}$sur les sites des trois observatoires. Le tracé des résidus contre les variables explicatives ne fait pas ressortir de forme particulière (comme un effet quadratique ou une tendance), signe que l'ajout de termes plus complexes n'est pas nécessaire. Leur répartition régulière permet de valider l'hypothèse d'homoscédasticité (la variance des erreurs est uniforme pour toutes les observations) et le tracé des autocorrélations montre l'absence de dépendance temporelle, validant l'hypothèse d'indépendance des résidus. Enfin, le tracé de la droite de Henri des résidus fait apparaître une assez bonne adéquation globale à la loi normale, tout en faisant ressortir une queue de distribution un peu plus épaisse pour les faibles valeurs des résidus. $\mathrm{Au}$ final, et avec ce bémol à propos de la normalité des résidus, les hypothèses de base des modèles de régression linéaire multiple sont bien respectées.

\section{Validation croisée}

Pour l'évaluation des modèles, la technique du « $k$-fold » de ré-échantillonnage de validation croisée a été mise en place. Dans notre cas, le nombre de blocs est de trois. Un exemple de résultat de la validation croisée est présenté en figure 5. La prévision en validation croisée est très proche de la prévision réalisée avec tout le jeu de données, ce qui montre une bonne capacité du modèle en généralisation. On observe aussi la difficulté du modèle à prévoir l'amplitude des pics de dépôts. entre les différentes projections divergent de manière sensible à partir de 2020-2030 (Hawkins et Sutton, 2009 ; Johns et al., 2011). Les variables de saisonnalité (indices cosinus et sinus) sont variables à l'échelle mensuelle, mais sans changements structurels dans le futur. Cependant, l'évolution climatique peut avoir des effets indirects comme des changements dans la pluviométrie, le rayonnement UV, dans les vitesses d'oxydation des polluants, le comportement des aérosols, etc. (Isaksen et al., 2009).

\section{Simulations climatiques}

Plusieurs simulations désagrégées, issues des travaux de Pagé et Terray (2010), ont été choisies pour reproduire l'évolution attendue des précipitations sur la France pour la période 20202040. La désagrégation est une méthode pour descendre à une échelle plus fine les simulations climatiques. Les scénarios d'émissions utilisés par les simulations Arpege V4, Arpege V4.5 Retic, CNCM33 et Hadgem2 sont les scénarios A1B. Les simulations utilisées sont issues de la désagrégation des simulations du modèle ArpegeClimat en version 4 et 4.5 (Déqué, 2007) et de la désagrégation des modèles atmosphériques de circulation générale du projet européen FP7-Ensembles (Brands et al., 2011) :

- Arpege V4 Cerfacs A1B M1 notée Arpege-A1B

- Arpege V4.5 Retic A1B notée Arpege 1

- CNCM33 membre 1

- Hadgem 2 membre 1 tion en $\mathrm{CO}_{2}$ de l'atmosphère ne cesse d'augmenter depuis 1950 et les écarts

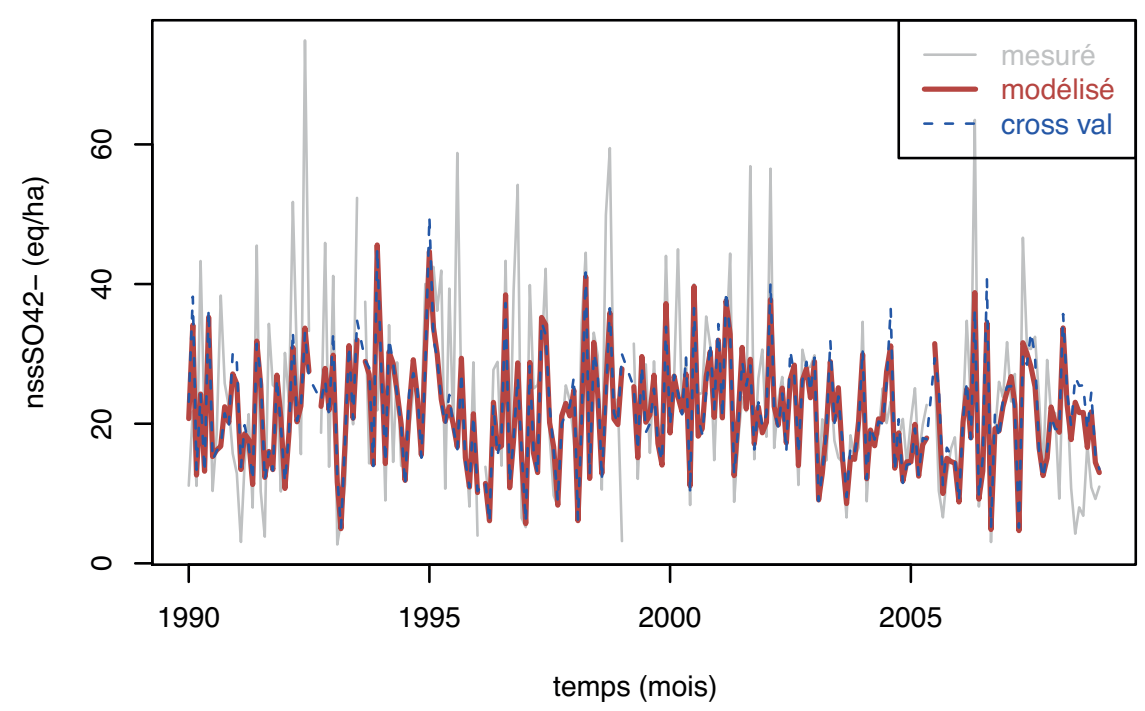

Figure 5. Prévision du modèle de régression linéaire multiple et du modèle de validation croisée comparée aux données initiales de dépôts mensuels de sulfate non marin au site WM08RE sur la période 1990-2008 $\left(R_{\mathrm{adj}}{ }^{2}=0,45 ; R_{\mathrm{adj}}{ }^{2}\right.$ est une version modifiée de $R^{2}$ qui a été ajustée pour le nombre de variables explicatives du modèle). 


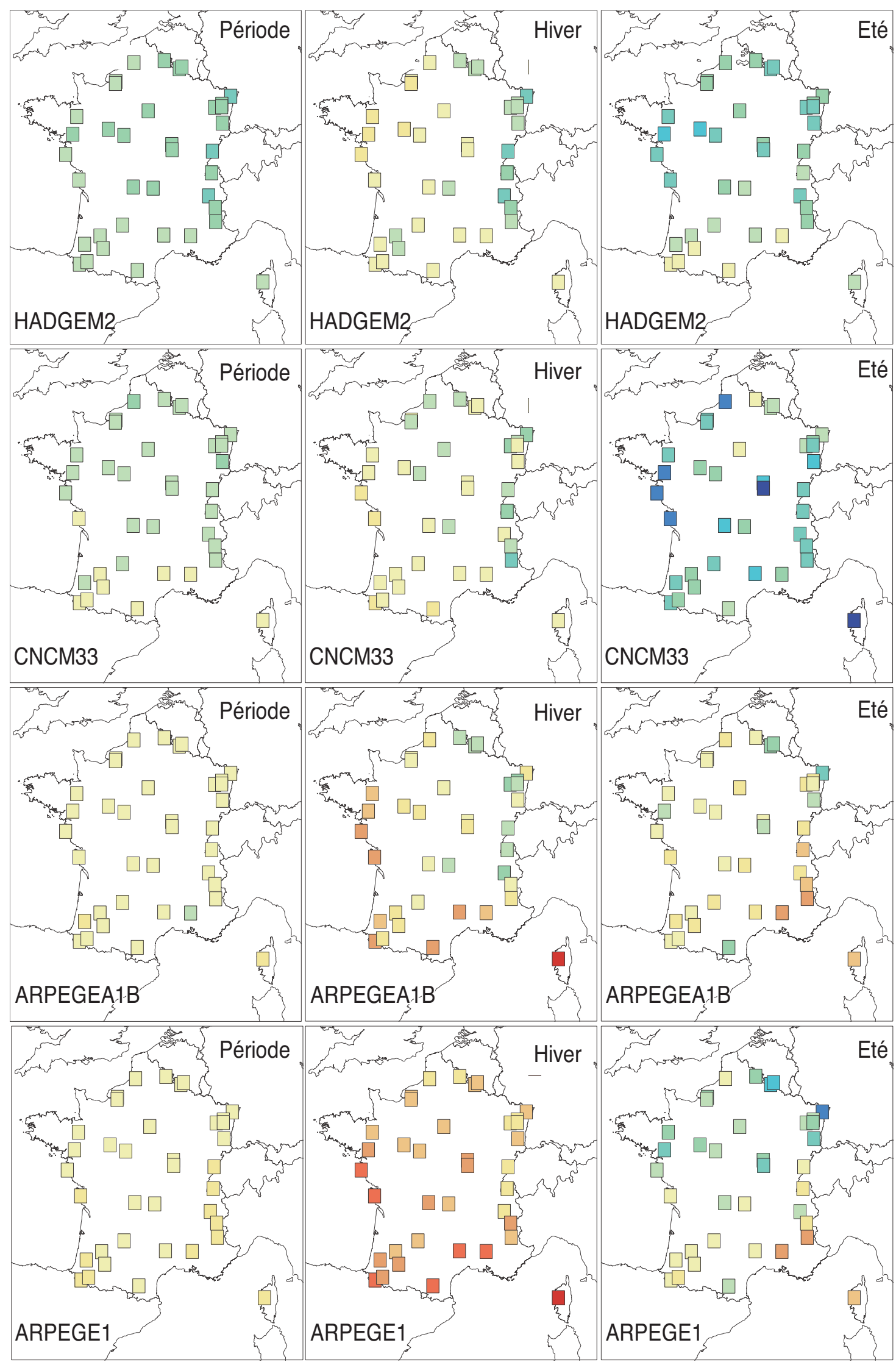

Changements projetés

\begin{tabular}{lrrr}
\hline $50 \%$ & $20 \% 30 \%$ & $0 \%-10 \%$ & $-30 \%-40 \%$ \\
$40 \% 50 \%$ & $10 \% 20 \%$ & $-10 \%-20 \%$ & $-40 \%-50 \%$ \\
$30 \% 40 \%$ & $0 \% 10 \%$ & $-20 \%-30 \%$ & $<-50 \%$
\end{tabular}

Figure 6. Changements projetés (\%) des précipitations totales moyennées sur la période 2020-2040 par rapport à la période 1990-2008, sur les mois d'hiver (décembre, janvier, février) et sur les mois d'été (juin, juillet, août) pour quatre simulations (Arpege1, Arpege-A1B, Hadgem2 et CNCM33) sur l'ensemble des sites utilisés dans cette étude. 
Ces quatre modèles contrastés ont été utilisés afin de représenter la plage d'incertitudes des simulations climatiques en utilisant un jeu réduit de modèles (Pagé et Terray, 2010). Par rapport à la période de référence de 19712000 sur la France et pour la période 2020-2040, certaines simulations sont caractérisées par un été relativement chaud et sec, c'est le cas du modèle Arpege1, ou chaud et humide dans le cas de Hadgem2. Pour les simulations (CNCM33 et Arpege-A1B), la période estivale ne montre pas de changement des quantités de précipitations, avec une augmentation modérée des températures. En période hivernale, CNCM33 simule des anomalies de précipitations aussi importantes que Hadgem2, mais avec des anomalies de température moins fortes pour CNCM33.

\section{Scénarios d'émissions}

Sur la base du programme Clean Air For Europe, la Commission européenne a adopté en 2005 une stratégie thématique ayant pour objectif la protection à long terme de la santé humaine et de l'environnement contre les effets de la pollution atmosphérique. Dans ce cadre, il a pu être établi différents scénarios d'évolution probable des émissions polluantes. Le scénario de référence utilisé dans cette étude, appelé Current Legislation, suppose la mise en œuvre intégrale de la législation existante sur la pollution de l'air et l'absence de législation supplémentaire. Les données de prévisions utilisées pour les émissions de l'Union Européenne sont disponibles avec le modèle GAINS-Europe online dans le groupe de scénarios TSAP_2012 (basé sur les résultats du projet EC4MACS). Dans les prochaines décennies, les prévisions indiquent que les émissions de $\mathrm{SO}_{X}$ vont être réduites drastiquement puisqu'il est envisagé une diminution de plus de $70 \%$ en 2030 par rapport à 2005 (Amann et al., 2012). Des baisses de plus de $65 \%$ en 2030 par rapport à 2005 seront aussi attendues dans les émissions de $\mathrm{NO}_{X}$, notamment concernant le trafic maritime (Amann et al., 2012). Les émissions de $\mathrm{NH}_{3}$ évolueraient substantiellement ; cependant, il y a de grandes différences dans la projection de l'évolution des émissions de $\mathrm{NH}_{3}$ entre les États membres (Skjoth et Geels, 2013). Par exemple, au Danemark, les émissions devraient diminuer, alors qu'en France les émissions en 2050 seraient du même ordre de grandeur qu'en 2005 (Amann et al., 2012).

\section{Résultats : projections à l'horizon 2020-2040}

\section{Pluviométrie}

Les anomalies entre les précipitations observées (1990-2008) et prévues (2020-2040) par les quatre simulations choisies ont été calculées sur l'ensemble des sites utilisés dans cette étude (figure 6). Sur la moyenne annuelle de la période 2020-2040, les changements de pluviométrie par rapport à la période 1990-2008 montrent une distribution spatiale particulière. On observe tout d'abord un contraste est-ouest. À l'inverse de la partie ouest, dans la partie est les précipitations annuelles augmentent avec un changement compris entre 0 et $20 \%$ sur Hadgem 2 et CNCM33. La deuxième caractéristique géographique, partagée par les simulations et plus modérément par Hadgem2, implique des changements dans le Sud méditerranéen et le Sud-Ouest. Pour ces deux zones, les précipitations annuelles diminuent pour 2020-2040, avec des changements compris entre $-10 \%$ et $-20 \%$.

Ces disparités régionales s'accentuent dans les projections des changements de la pluviométrie saisonnière (figure 6). Sur les reliefs des Alpes, du Massif central, du Jura, des Vosges et des Ardennes, les simulations prévoient en hiver une augmentation de pluviométrie (entre 0 et $30 \%$ ), mise à part la simulation Arpege1. Sur les façades maritimes du territoire, un assèchement est généralisé en hiver pour toutes les simulations avec des pourcentages plus forts et supérieurs à $50 \%$ pour Arpege 1 . Ces projections sont en accord avec les résultats de Boé et al. (2009) qui prévoient que, sur la période 2046-2065 par rapport à la climatologie 1971-2000, les changements de pluviométrie seront marqués, en hiver, par un fort contraste spatial, entre le sud-est et le sud-ouest de la France. En été, une diminution des précipitations sur les reliefs est indiquée par les simulations Arpege-A1B et Arpege 1 (figure 6). Les résultats des modèles Hagdem2 et CNCM33 suggèrent une augmentation des précipitations quasi généralisée sur le territoire et de façon plus marquée sur la pointe bretonne.

\section{Chimie des dépôts atmosphériques}

Avec le modèle statistique de régression linéaire présenté plus haut, les données passées de dépôts de sulfate non marin (nss $\mathrm{SO}_{4}^{2-}$ ), de nitrate $\left(\mathrm{NO}_{3}^{-}\right)$et d'ammonium $\left(\mathrm{NH}_{4}^{+}\right)$ont été reconstruites en utilisant les émissions issues des activités anthropiques et les précipitations issues des données Safran à proximité des stations des trois observatoires nationaux. Le scenario d'émission TSAP-2012 et les quatre simulations de projections climatiques ont été utilisées entre 2020-2040 (Arpege1, ArpegeA1B, Hadgem2, CNCM33). Les scénarios plus ou moins secs et plus ou moins pluvieux donnent des résultats similaires en matière de changements projetés sur les dépôts. Une moyenne des résultats de simulation des quatre scénarios a donc été utilisée pour tenir compte de l'évolution possible future de la chimie des dépôts atmosphériques issue des quatre simulations.

\section{Changements annuels projetés}

La figure 7 représente les changements annuels projetés, entre 2020-2040, par la moyenne multisimulations $(n=4)$ des dépôts projetés de nnss $\mathrm{SO}_{4}^{2-}, \mathrm{NO}_{3}^{-}$et $\mathrm{NH}_{4}^{+}$sur les sites des observatoires, en référence avec les dépôts modélisés entre 1990 et 2008. Les diminutions projetées sont fortes et homogènes pour les dépôts de $\mathrm{nsSSO}_{4}^{2-}$ comprises entre $-45 \%$ et $+1 \%$. Les changements projetés pour les dépôts de $\mathrm{NO}_{3}^{-}$ne sont pas homogènes, une baisse pour 25 stations en moyenne de $21 \%$ et une augmentation pour 12 en moyenne de $23 \%$. Les changements projetés des dépôts de $\mathrm{NH}_{4}^{+}$sont faibles et sur de nombreux sites, ils sont prévus à la baisse, comprise entre $25 \%$ et $2 \%$.

Sur les dépôts, le motif déterminant des évolutions futures est dépendant du lien actuel entre les dépôts et les émissions polluantes. Dans le passé, malgré une réduction globale des émissions, les dépôts de $\mathrm{NO}_{3}^{-}$ont pu augmenter sur certains sites. Plusieurs hypothèses peuvent être avancées : le rôle des oxydes d'azote dans le budget d'oxydants atmosphériques (Pun et Seigneur, 2001) ; l'implication des émissions d'autres polluants (tels que $\mathrm{SO}_{2}, \mathrm{NH}_{3}$ et les composés organiques volatils) et le rôle du nitrate de peroxyacétyle (PAN) dans la formation de $\mathrm{NO}_{3}^{-}$(Stockwell et al., 2003). La modélisation statistique reproduit cette non-linéarité entre émissions et dépôts de $\mathrm{NO}_{3}^{-}$. Malgré la réduction des émissions de $\mathrm{NO}_{x}$, il n'y aurait pas de diminution nette globale des dépôts de $\mathrm{NO}_{3}{ }^{-}$à l'horizon 2020-2040.

En raison du poids dans les régressions de la variable pluviométrique (coefficient de régression égal à 0,644 par exemple au site WM08RE, cf. équation 2), il apparaît que la précipitation est le 

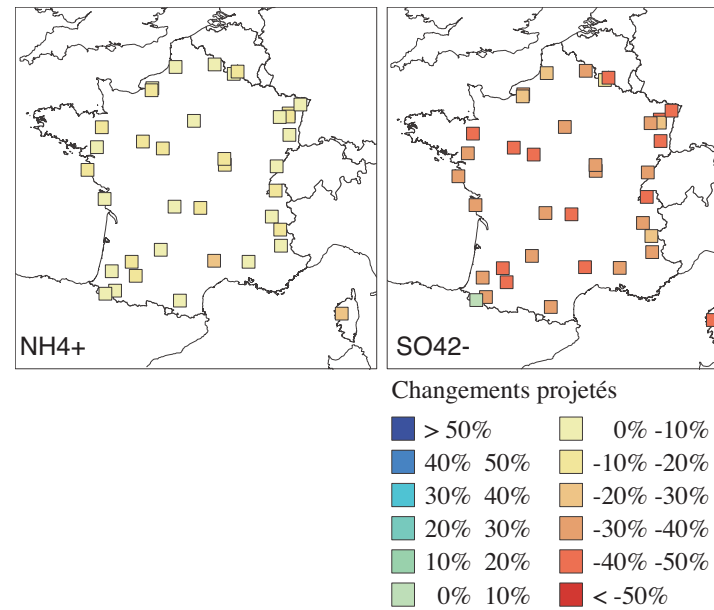

Figure 7. Changements projetés des dépôts annuels d'ammonium $\left(\mathrm{NH}_{4}^{+}\right)$, de sulfate non marin $\left(\mathrm{SO}_{4}^{2-}\right)$ et de nitrate $\left(\mathrm{NO}_{3}{ }^{-}\right)$selon la moyenne multi-modèles entre 2020-2040, par rapport à la période 1990-2008 sur l'ensemble des sites utilisés dans cette étude. facteur d'influence majoritaire dans la modélisation statistique des dépôts ioniques. Cependant, l'influence des émissions n'est pas négligeable, comme l'illustre la différence d'amplitude des changements des dépôts de $\mathrm{NH}_{4}{ }^{+}$et de ceux de $\mathrm{nsSSO}_{4}^{2-}$. Alors que les changements annuels projetés des dépôts de $\mathrm{nssSO}_{4}^{2-}$ peuvent être proches de $-50 \%$, ceux de $\mathrm{NH}_{4}{ }^{+}$ne dépassent pas les $-20 \%$. Ceci est principalement dû aux écarts dans les scénarios d'émission qui prévoient des changements importants pour $\mathrm{SO}_{x}$ et des changements faibles pour $\mathrm{NH}_{3}$, respectivement $-70 \%$ et $+1,8 \%$ entre 2005 et 2030 .

\section{Changements saisonniers projetés}

Les écarts saisonniers de dépôts de nssSO ${ }_{4}^{2-}, \mathrm{NH}_{4}{ }^{+}$et $\mathrm{NO}_{3}{ }^{-}$entre le temps présent et les simulations futures ont été analysés sur les stations selon les quatre simulations climatiques sélectionnées. La figure 8 représente la variation temporelle des dépôts de $\mathrm{NO}_{3}{ }^{-}$sur 28 jours des précipitations et des dépôts aux temps présent et futur simulé sur le site BC05CR, choisi pour son anomalie positive $(+25,6 \%)$ en dépôts de $\mathrm{NO}_{3}{ }^{-}$. Aujourd'hui, les précipitations sur ce site présentent une variation bimodale avec un pic de précipitation au printemps et un autre plus intense en automne, les maxima de dépôts interviennent au printemps et à la fin de l'été. Dans le cas du futur 2020-2040, les précipitations simulées par Hadgem2 et CNCM33 seraient plus intenses au printemps et en fin d'été, respectivement. Une augmentation des précipitations forcerait nettement le dépôt à l'augmentation sur ces périodes. La courbe bimodale des dépôts serait conservée sous simulation CNCM33 et Arpege1, alors que la courbe bimodale des précipitations n'existerait pas sous simulation CNCM33. Ainsi, au printemps, le pic des dépôts ne serait pas contrôlé par les précipitations dans cette simulation. Dans ce cas précis, le changement dans les émissions anthropiques pourrait avoir une influence plus marquée que les précipitations.

Les résultats sur le site WM67DO sont présentés dans la figure 9 , choisi pour ses anomalies négatives $(-28,7 \%)$ de dépôts annuels de $\mathrm{nsSSO}_{4}^{2-}$ en 2020-2040 par rapport à 1990-2008. Les quatre modèles s'accordent en moyenne sur une diminution des précipitations en hiver et une augmentation sur la fin de l'été. Les dépôts de $\mathrm{nssSO}_{4}^{2-}$ qui actuellement peuvent varier du simple au double entre l'hiver et le printemps, sont simulés à la baisse sur cette période et présenteraient un pic, non plus printanier, mais estival, suivant celui des précipitations. L'influence de la tendance dans les émissions serait dans ce cas plus marquée.

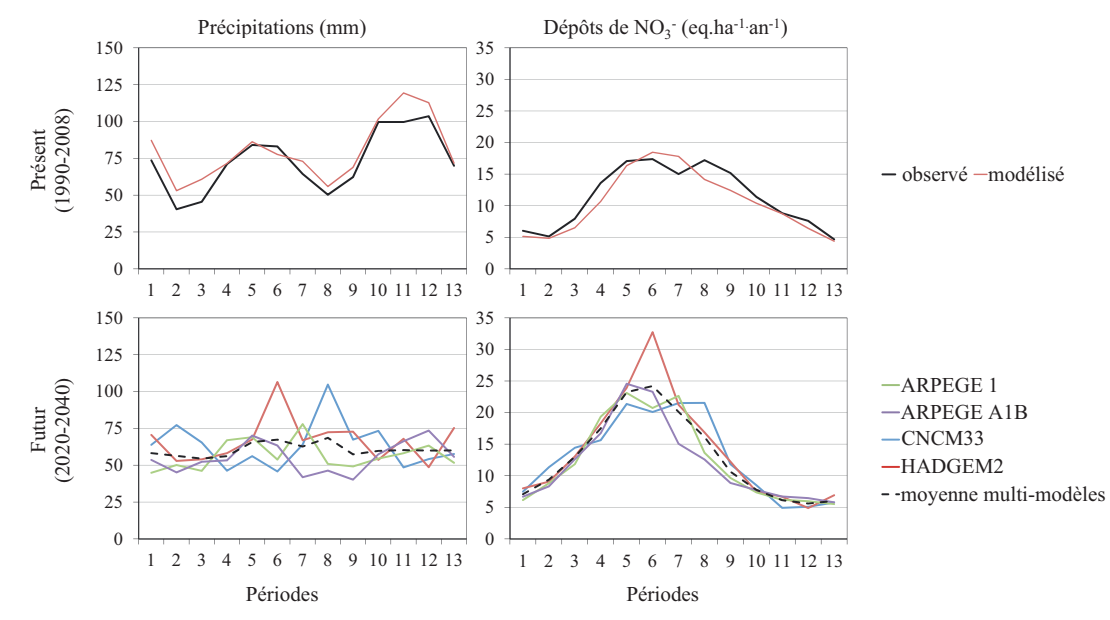

Figure 8. Profils périodiques (28 jours) des changements projetés sur la station BC05CR des précipitations, des dépôts de $\mathrm{NO}_{3}^{-}$selon la moyenne multi-modèles entre 2020-2040 par rapport à la période 1990-2008.
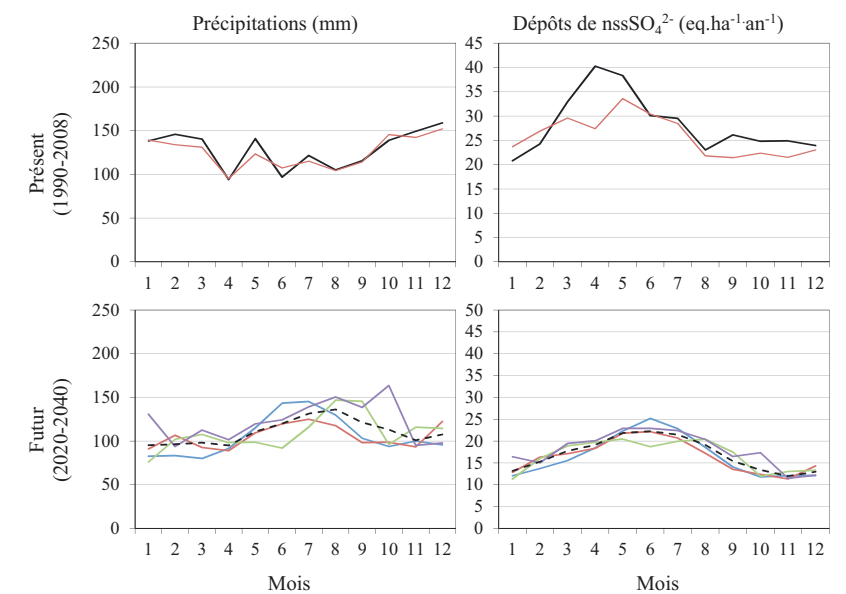

-observé -modélisé

Figure 9. Profils mensuels des changements projetés sur la station WM67D0 des précipitations, des dépôts de $\mathrm{nsSSO}_{4}^{2-}$ selon la moyenne multi-modèles entre 2020-2040 par rapport à la période 1990-2008. 


\section{Discussion}

À ces prédictions de dépôts de nss $\mathrm{SO}_{4}^{2-}, \mathrm{NO}_{3}^{-}$et $\mathrm{NH}_{4}^{+}$sont associées des incertitudes de différentes provenances.

En premier lieu, il y a l'incertitude liée à la capacité du modèle statistique à reproduire les mesures de dépôts. Cette incertitude est le résultat du choix du type de modèle, des variables, dont certaines, très déterminantes, peuvent être manquantes. Dans la mesure où ces variables ne sont pas corrélées entreelles ou à d'autres variables explicatives, la température et l'humidité relative pourraient être utiles à la modélisation. De plus, l'incertitude du modèle statistique est reliée à la qualité de la pluviométrie modélisée issue de l'analyse Safran. En effet, les corrélations peuvent être faibles entre ces modélisations et les observations pluviométriques sur certains sites, notamment en zone montagneuse. Les précipitations sont difficiles à modéliser, car leurs processus de formation intègrent des processus non linéaires à très petite échelle. L'utilisation de ces précipitations modélisées était néanmoins indispensable pour pouvoir s'affranchir de ce biais dans les prévisions de dépôts.

En second lieu, à cette incertitude du modèle statistique, il faut associer les nombreuses autres sources d'incertitudes, depuis la métrologie des dépôts atmosphériques jusqu'aux représentations dans les modèles climatiques de la physique des phénomènes. Il faut également ajouter les incertitudes liées aux scénarios d'émissions de gaz à effet de serre, à la prise en compte des facteurs socio-économiques et aux éventuelles rétroactions.

Concernant, les modèles climatiques, la modélisation des nuages et le rôle des aérosols ont été identifiés comme des sources majeures d'incertitudes dans les travaux à l'échelle globale. Ces sources d'incertitudes des simulations climatiques ont été étudiées en détail par le Groupe d'experts intergouvernemental sur l'évolution du climat (IPCC, 2013). Pour pouvoir utiliser ces simulations dans des études d'impacts, la méthode de descente d'échelle ajoute une nouvelle source d'incertitude. La désagrégation statistique s'appuie sur la circulation à grande échelle et sur les types de temps associés (Pagé et al., 2009). Cette méthode est fondée sur une hypothèse forte, celle de la conservation des liens physiques entre les variables locales climatiques à prévoir et la circulation atmosphérique à grande échelle, entre le climat présent et le climat perturbé. Pour les précipitations, Quintana-Segui et al. (2010) ont indiqué que les méthodes de descente d'échelle produisent des tendances similaires sur le long terme, mais que, spatialement, les différences peuvent être importantes, en particulier dans la zone méditerranéenne, les zones côtières et les zones montagneuses, d'autant plus que ces différences dépendent des saisons.

S'agissant des émissions de gaz à effet de serre, les scénarios fondés sur le forçage radiatif des différents composants atmosphériques Representative Concentration Pathway (RCP) ont été couplés à des projections socioéconomiques (SSP) lors de la phase 6 du projet d'intercomparaison de modèles couplés - CMIP6. L'utilisation de plusieurs modèles est, à ce jour, le meilleur moyen de rendre compte des incertitudes sur les modèles. Par conséquent, l'utilisation de plusieurs modèles d'émissions anthropiques serait aussi intéressante pour évaluer les incertitudes sur cette composante.

De nombreuses études ont été réalisées sur le devenir de la composition chimique des dépôts atmosphériques dans un contexte de changement climatique à une échelle locale et globale (Hole, 2008 ; de Vries et Posch, 2011). Ces études ont montré la nécessité de prendre en compte l'influence des tendances des précipitations, liées aux évolutions climatiques synoptiques, sur celles de la composition chimique des dépôts atmosphériques. En outre, il est important de coupler les évolutions sur la pluviométrie avec les changements de température et les changements d'émissions anthropiques de polluants. Skjoth et al. (2013) indiquent que lors d'un changement climatique les effets de la température sur les émissions d'ammoniac sont plus prononcés. Hole et Engardt (2008) ont montré que les dépôts secs d'ammonium augmenteraient sur la période 2021-2050, par rapport à la période 1961-1990, potentiellement sous l'effet d'une augmentation des surfaces humides et d'une augmentation de la volatilisation de l'ammoniac.

\section{Conclusion}

La complexité du système physicochimique atmosphérique limite l'aptitude des modèles déterminants de chimie-transport à prévoir (dans le temps présent et dans le futur) la chimie des dépôts atmosphériques. Une approche complémentaire a été conçue dans ces travaux à partir de longues séries de mesures issues de trois observatoires français de surveillance de la pollution de fond. Un modèle statistique a été développé à partir de variables explicatives (émissions polluantes, précipitations et indices saisonniers) pour reproduire les dépôts soufrés et azotés collectés sur 37 stations des observatoires nationaux. La construction de ce modèle a été consolidée sur l'historique des données pour pouvoir envisager des prévisions. À l'horizon 2020-2040, les émissions polluantes ont été estimées à partir du scénario $\mathrm{A} 1 \mathrm{~B}$ et les précipitations à partir de quatre simulations climatiques Arpege-A1B, Arpege1, Hadgem2 et CNCM33. En combinant simultanément les émissions polluantes et les précipitations à l'horizon 2020-2040, le modèle statistique prévisionnel a permis d'appréhender les changements projetés de la composition chimique des dépôts atmosphériques. Les résultats obtenus montrent que les dépôts de $\mathrm{nsSSO}_{4}^{2-}$ et de $\mathrm{NH}_{4}{ }^{+}$ sont susceptibles de diminuer (respectivement en moyenne de $35 \%$ et de $11 \%$ ), mais ceux de $\mathrm{NO}_{3}^{-}$pourront augmenter ou diminuer selon des disparités régionales (en moyenne $-7 \%$ ). Le changement des variabilités saisonnières des pluviométries pourrait forcer les dépôts à l'augmentation. Ce travail montre tout l'intérêt des données d'observation sur le long terme, que ce soient les variables climatiques ou la composition chimique des dépôts atmosphériques pour une meilleure compréhension des liens pollution atmosphérique-climat et pour l'évaluation des politiques de réduction des émissions polluantes. Le modèle statistique pourrait notamment être réutilisé en post-traitement statistique pour améliorer des modélisations déterministes de chimie-transport.

\section{Remerciements}

Ce travail a été rendu possible par le soutien financier de l'Ademe et le ministère français en charge de l'environnement par le biais du programme Primequal/Predit2 (contrat $\mathrm{n}^{\circ}$ 0962C0014). Les auteurs sont reconnaissants envers les gestionnaires des stations des observatoires ainsi qu'envers le personnel du laboratoire d'analyse et remercient Météo-France et l'Office national des forêts. 


\section{Bibliographie}

Amann M., Borken-Kleefeld J., Cofala J., Heyes C.J., Klimont Z., Rafaj P., Purohit P., Schopp W., Winiwarter W., 2012. Future emissions of air pollutants in Europe - Current Legislation baseline and the scope for further reductions. TSAP Report 1, International Institute for Applied Systems Analysis, Laxenburg, Austria, 62 p.

Barthod C., 1994. Le système de surveillance de l'état sanitaire de la forêt en France. Rev. For. Fr., 46, 564-571.

Boé J., Terray L., Martin E., Habets F., 2009. Projected changes in components of the hydrological cycle in French river basins during the 21 st century. Water Resource Res., 45, W08426. doi: 10.1029/2008wr007437

Brands S., Herrera S., San-Martín D., Gutiérrez J., 2011. Validation of the ENSEMBLES global climate models over southwestern Europe using probability density functions, from a downscaling perspective. Clim. Res. , 48, 145-161. doi: 10.3354/cr00995

Carmichael G., Wild 0., 2010. Global and regional modelling (Dentener F., Keating T., Akimoto H., eds) Hemispheric Transport of Air Pollution 2010: Part a: Ozone and Particulate Matter. Air Pollution Studies. United Nations Publications, Geneva, 135-198.

Cénac N., Zéphoris M., 1992. Une décennie de surveillance de l'acidité des précipitations en France. Ministère de l'Équipement, du Logement et des Transports, Direction de la Météorologie nationale, Trappes, 39 p.

Chattopadhyay G., Chattopadhyay S., Chakraborthy P., 2012. Principal component analysis and neurocomputing-based models for total ozone concentration over different urban regions of India. Theor. Appl. Climatol., 109, 221-231. doi: 10.1007/s00704-011-0569-7

De Vos T., Zhang L., 2012. High resolution mapping of total deposition of acidifying pollutants. Atmos. Environ., 57, 80-90. doi: 10.1016/j.atmosenv.2012.04.037

De Vries W., Posch M., 2011. Modelling the impact of nitrogen deposition, climate change and nutrient limitations on tree carbon sequestration in Europe for the period 1900-2050. Environ. Pollut., 159, 2289-2299. doi: 10.1016/j.envpol.2010.11.023

Déqué M., 2007. Frequency of precipitation and temperature extremes over France in an anthropogenic scenario: Model results and statistical correction according to observed values. Global Planet. Change, 57, 16-26. doi: 10.1016/j.gloplacha.2006.11.030

Emep/Ceip 2012. Emission data reported to CLRTAP in 2012. National total without S11. \#Emission data reported to CLRTAP in 2012 \#Missing data completed by CEIP, CEIP2012 (2012-04-12).

Hawkins E., Sutton R., 2009. The potential to narrow uncertainty in regional climate predictions. Bull. Am. Meteorol. Soc., 90, 1095-1107. doi: 10.1175/2009bams2607.1 Hole L., Engardt M., 2008. Climate change impact on atmospheric nitrogen deposition in northwestern Europe: a model study. Ambio, 37, 9-17. doi: 10.1579/00447447(2008)37[9:ccioan]2.0.c0;2

Hulme M., Barrow E.M., Arnell N.W., Harrison P.A., Johns T.C., Downing T.E., 1999. Relative impacts of human-induced climate change and natural climate variability. Nature, 397, 688-691. doi: 10.1038/17789

IPCC, 2013. Climate Change 2013: The Physical Science Basis. Contribution of Working Group I to the Fifth Assessment Report of the Intergovernmental Panel on Climate Change. Cambridge University Press, Cambridge, UK, and New York, NY, USA., 1535 p.

Isaksen I.S.A., Granier C., Myhre G., Berntsen T.K., Dalsoren S.B., Gauss M., Klimont Z., Benestad R., Bousquet P., Collins W., Cox T., Eyring V., Fowler D., Fuzzi S., Jöckel P., Laj P., Lohmann U., Maione M., Monks P., Prevot A.S.H., Raes F., Richter A., Rognerud B., Schulz M., Shindell D., Stevenson D.S., Storelvmo T., Wang W.C., Van Weele M., Wild M., Wuebbles D., 2009. Atmospheric composition change: climate-chemistry interactions. Atmos. Environ., 43, 5138-5192. doi: 10.1016/j.atmosenv.2009.08.003

Johns T., Royer J.F., Höschel I., Huebener H., Roeckner E., Manzini E., May W., Dufresne J.-L., Otterå O., van Vuuren D., Salas y Melia D., Giorgetta M., Denvil S., Yang S., Fogli P., Körper J., Tjiputra J., Stehfest E., Hewitt C., 2011. Climate change under aggressive mitigation: the ENSEMBLES multi-model experiment. Clim. Dyn., 37, 1975-2003. doi: 10.1007/s00382-011-1005-5

Kohler A., 1980. The WMO BAPMoN. Special Environmental Report, No. 14, World Meteorological Organisation, 207-215.

Landmann G., 1991. Les recherches en France sur le dépérissement. Programme DEFORPA 2. Nancy, Engref, 120 p.

Makridakis S., Wheelwright S.C., Hyndman R.J., 1998. Forecasting: Methods and Applications. John Wiley \& Sons, Inc., 642 p.

Moustris K.P., Nastos P.T., Larissi I.K., Paliatsos A.G., 2012. Application of multiple linear regression models and artificial neural networks on the surface ozone forecast in the greater Athens area, Greece. Adv. Meteorol., 2012, 1-8. doi: 10.1155/2012/894714

Muller M., 1984. Livre blanc sur les pluies acides : première approche scientifique du problème en France. Republique française, Secrétariat d'État à l'Environnement et à la Qualité de Vie, Service de la recherche, des études et du traitement de l'information sur l'environnement, Direction de la prévention des pollutions, 253 p.

Nickerson D.M., Madsen B.C., 2005. Nonlinear regression and ARIMA models for precipitation chemistry in East Central Florida from 1978 to 1997. Environ. Pollut., 135, 371 379. doi: 10.1016/j.envpol.2004.11.010

Pagé C., Terray L., 2010. Nouvelles projections climatiques à échelle fine sur la France pour le 21ème siècle : les scénarii SCRATCH2010. Technical Report TR/CMGC/10/58. SUC au Cerfacs, URA Cerfacs/CNRS No 1875CS. Toulouse, France, 25 p.

Pagé C., Terray L., Boé J., 2009. DSclim: a software package to downscale climate scenarios at regional scale using a weather-typing based statistical methodology. Report TR/CMGC/09/21. Toulouse, France, SUC au Cerfacs, URA Cerfacs/CNRS No 1875, 60 p.

Pun B.K., Seigneur C., 2001. Sensitivity of particulate matter nitrate formation to precursor emissions in the California San Joaquin Valley. Environ. Sci. Technol., 35, 29792987. doi: 10.1021/es0018973

Quintana-Segui P., Le Moigne P., Durand Y., Martin E., Habets F., Baillon M., Canellas C., Franchisteguy L., Morel S., 2008. Analysis of near-surface atmospheric variables: validation of the SAFRAN analysis over France. J. Appl. Meteorol. Climatol., 42, 92-107. doi: 10.1175/2007JAMC1636.1

Quintana-Segui P., Ribes A., Martin E., Habets F., Boé J., 2010. Comparison of three downscaling methods in simulating the impact of climate change on the hydrology of Mediterranean basins. J. Hydrol., 383, 111-124. doi: 10.1016/j.jhydrol.2009.09.050

Sauvage S., Plaisance H., Locoge N., Wroblewski A., Coddeville P., Galloo J., 2009. Long term measurement and source apportionment of non-methane hydrocarbons in three French rural areas. Atmos. Environ., 43, 2430-2441. doi: 10.1016/j.atmosenv.2009.02.001

Schucht S., Colette A., Rao S., Holland M., Schopp W., Kolp P., Klimont Z., Bessagnet B., Szopa S., Vautard R., Brignon J. M., Rouil L., 2015. Moving towards ambitious climate policies: Monetised health benefits from improved air quality could offset mitigation costs in Europe. Environ. Sci. Policy, 50, 252-269. doi: 10.1016/j.envsci.2015.03.001

Skjoth C.A., Geels C., 2013. The effect of climate and climate change on ammonia emissions in Europe. Atmos. Chem. Phys., 13, 117-128. doi: 10.5194/acp-13-117-2013 Stockwell W.R., Kuhns H., Etyemezian V., Green M.C., Chow J.C., Watson J.G., 2003. The Treasure Valley secondary aerosol study II: modeling of the formation of inorganic secondary aerosols and precursors for southwestern Idaho. Atmos. Environ. , 37, 525-534. doi: 10.1016/S1352-2310(02)00895-6

Uggerud H.T., Hjellbrekke A.G., 2011. The twenty-seventh and twenty-eighth intercomparison of analytical methods within EMEP. NILU Report EMEP/CCC-Report, 110 p.

Ulrich E., Lelong N., Lanier M., Schneider A., 1998. Regional differences in the relation between monthly precipitation and bulk concentration in France (Renecofor). Water, Air, Soil Pollut., 102, 239-257. doi: 10.1023/A:1004944828649

Vet R., Artz R.S., Carou S., Shaw M., Ro C.-U., Aas W., Baker A., Bowersox V.C., Dentener F., Galy-Lacaux C., Hou A., Pienaar J.J., Gillett R., Forti M.C., Gromov S., Hara H., Khodzher T., Mahowald N.M., Nickovic S., Rao P.S.P., Reid N.W., 2014. A global assessment of precipitation chemistry and deposition of sulfur, nitrogen, sea salt, base cations, organic acids, acidity and pH, and phosphorus. Atmos. Environ., 93, 3-100. doi: 10.1016/j.atmosenv.2013.10.060 